\title{
Genome-wide identification of the PEBP genes in pears and the putative role of $P b F T$ in flower bud differentiation
}

\author{
Shuliang Zhao ${ }^{1}$, Yarui Wei ${ }^{1}$, Hongguang Pang ${ }^{1}$, Jianfeng Xu ${ }^{1}$, Yingli Li ${ }^{1}$, Haixia Zhang ${ }^{1}$, Jianguang Zhang ${ }^{\text {Corresp., }}{ }^{1}$, \\ Yuxing Zhang Corresp. 1 \\ ${ }^{1}$ College of Horticulture, Hebei Agricultural University, Baoding, Hebei, China \\ Corresponding Authors: Jianguang Zhang, Yuxing Zhang \\ Email address: yyzjg@hebau.edu.cn, zhyx@hebau.edu.cn
}

Although Phosphatidylethanolamine-binding protein (PEBP) genes have been identified in several plants, little is known about PEBP genes in pears. In this study, a total of 24 PEBP genes were identified, in which 10, 5 and 9 were from Pyrus bretschneideri genome, Pyrus communis genome and Pyrus betuleafolia genome, respectively. Subsequently, gene structure, phylogenetic relationship, chromosomal localization, promoter regions, collinearity and expression were determined with these PEBP genes. It was found that only PbFT from PEBP genes of Pyrus bretschneideri was relatively highly expressed in leaves during flower bud differentiation. Whereas, expression patterns of TFL1 homologues, gene23124 and gene16540, were different from PbFT in buds. A trail of different treatments indicated that PbFT expressions in leaves were regulated by day-length and circadian clock, which was completely different from many other scholars' findings. Additionally, the phenotype of transgenic Arabidopsis suggested that PbFT played a role in not only promoting flower bud differentiation, but also regulating the balance between vegetative and reproductive growth. These results may provide important information for further understanding of the evolution and function of PEBP genes in pears. 


\section{Genome-wide identification of the PEBP genes in}

3 pears and the putative role of $P b F T$ in the flower bud

4 differentiation

5

6

7

8

9

10

11

12

13

14

15

16

17

18

19

20

21

22

23

24

25

26

27

28

29

30

31

32

33

34

35

36

37

38

Shuliang Zhao, Yarui Wei, Hongguang Pang, Jianfeng Xu, Yingli Li, Haixia Zhang, Jianguang Zhang* and Yuxing Zhang*

College of Horticulture, Hebei Agricultural University, Baoding, Hebei, China

Corresponding Author:

Jianguang Zhang and Yuxing Zhang

Leka Street, Baoding, Hebei, 071000, China

Email address: yyzjg@hebau.edu.cn; zhyx@hebau.edu.cn

\section{Abstract}

Although Phosphatidylethanolamine-binding protein (PEBP) genes have been identified in several plants, little is known about $P E B P$ genes in pears. In this study, a total of $24 P E B P$ genes were identified, in which 10, 5 and 9 were from Pyrus bretschneideri genome, Pyrus communis genome and Pyrus betuleafolia genome, respectively. Subsequently, gene structure, phylogenetic relationship, chromosomal localization, promoter regions, collinearity and expression were determined with these PEBP genes. It was found that only PbFT from PEBP genes of Pyrus bretschneideri was relatively highly expressed in leaves during flower bud differentiation. Whereas, expression patterns of TFL1 homologues, gene23124 and gene 16540, were different from $P b F T$ in buds. A trail of different treatments indicated that $P b F T$ expressions in leaves were regulated by day-length and circadian clock, which was completely different from many other scholars' findings. Additionally, the phenotype of transgenic Arabidopsis suggested that $\mathrm{PbFT}$ played a role in not only promoting flower bud differentiation, but also regulating the balance between vegetative and reproductive growth. These results may provide important information for further understanding of the evolution and function of $P E B P$ genes in pears.

\section{Introduction}

Phosphatidylethanolamine-binding proteins (PEBPs) are named for their evolutionarily conserved phosphatidylethanolamine-binding domains and widely found in plants, animals and yeast (Chautard et al. 2004a; Leeggangers et al. 2018; Sun et al. 2018). In plants, the PEBP genes family is mainly divided into 3 subfamilies, FLOWERING LOCUS T (FT) -like, TERMINAL FLOWER 1 (TFL1) -like and MOTHER OF FT AND TFL1 (MFT) -like (Li et al. 
2015). MFT-like is the ancestor of the $F T$-like and TFL1-like subfamilies, and is not found in moss and lycopodium. FT/TFL1 homologous genes appeared along with the evolution of seed plants. FT/TFL1-like gene plays an important role in the transformation process of seed plants from vegetative to reproductive growth (Karlgren et al. 2011).

Many $P E B P$ family members have been identified in several plant species, including Arabidopsis (Peng et al. 2015), maize (Danilevskaya et al. 2008), cotton (Wang et al. 2018), soybean (Zhang et al. 2015), tulip (Leeggangers et al. 2018), poplar (Igasaki et al. 2008) and kiwifruit (Voogd et al. 2017). In Arabidopsis, the PEBP family contains 6 gene members, $F T$, TFL1, TWIN SISTER OF FT (TSF), BROTHER OF FT AND TFL1 (BFT), ARABIDOPSIS THALIANA CENTRORADIALIS (ATC), and MFT(Peng et al. 2015). FT encodes a signaling protein (or mRNA) called florigen, which serves as a long-distance signal to induce flowering (Putterill \& Varkonyi-Gasic 2016). Another protein encoded by TFL1 whose sequence is closely related to $F T$, performs the opposite function to repress flowering(Baumann et al. 2015). Despite extensive sequence conservation, $P E B P$ genes can act as regulators of various signaling pathways to control growth and differentiation (Chautard et al. 2004b; Yeung et al. 1999). At present, identification of PEBP genes becomes more important in plant genomes. Pear (Pyrus spp.) is one of the most important fruit crops in the world. $P E B P$ genes of pears have not been systematically analyzed, so that the biological functions of $P E B P$ genes in pears have remained unclear until now. In this study, the $P E B P$ genes in pears genomes were identified and analyzed comparatively, including gene phylogeny, chromosomal locations, protein conserved domains, and cis-elements of their promoters. A phylogenetic analysis was also performed using PEBP genes searched from European pear, a wild pear and Arabidopsis. Moreover, we tentatively revealed the expression patterns of $P E B P$ genes and functions of $P b F T$ in pears during the flower bud differentiation. The results may provide a solid foundation to understand the distribution, structure and evolution of the $P E B P$ genes in pears, and will contribute to investigating the detailed functional differentiation and applying these genes in the future.

\section{Materials \& Methods}

\section{Search for PEBP Genes in Pears}

The genome sequences of 'Dangshan Suli' (Pyrus bretschneideri), 'Bartlett' (Pyrus communis) and 'Shanxi Duli' (Pyrus betuleafolia) were downloaded from the Genome Database for Rosaceae (GDR) (http://www.rosaceae.org/). The PEBP genes information of Arabidopsis was downloaded from the Arabidopsis Information Resource (TAIR) (http://www.arabidopsis.org/). To identify PEBP genes, the PEBP-domain profile (PF01161) was obtained from the Pfam database (http://pfam.xfam.org/). The hidden Markov models (HMM) profiles were used to search against the pear protein sequence data using default parameters and removed the nonrepresentative transcripts(Johnson et al. 2010). Then, the remaining sequences were checked for the conserved PEBP domain using Pfam(Finn et al. 2016) and SMART (http://smart.emblheidelberg.de/).

\section{Protein Properties and Sequence Analysis}


The pear PEBP sequences were uploaded to ExPASy (http://web.expasy.org/protparam/) to calculate the number of amino acids, molecular weights and isoelectric points (pI).

\section{Phylogenetic Tree Construction, Gene Structure and Protein Motif Analysis}

The phylogenetic tree was constructed for the sequences of PEBP, using MEGA v6.0 (Tamura et al. 2013) by the Neighbor-Joining (NJ) method with 1000 bootstrap replicates. The PEBP gene structures were obtained by alignment of open reading frames (ORFs) with corresponding genomic sequences along with the Gene Structure Display Server(Hu et al. 2014). Sequence logos of domain alignments were created using the MEME (http://meme-suite.org/tools/meme) with the default settings, except that the minimum and maximum motif widths were set to 10 and 60 amino acids, respectively. PEBP of both pears and Arabidopsis were analyzed.

\section{Physical Localization of PEBP Genes on Chromosomes and Synteny Analysis}

To identify chromosomal location of $P E B P$ genes, the genome annotation data was collected and mapped with TBtools(Chen et al. 2018). Synteny analysis of PEBP genes was performed by using TBtools.

\section{Calculation of Ks and Ka}

The synonymous substitution (Ks) and non-synonymous substitution $(\mathrm{Ka})$ rates were calculated using DnaSP v5(Librado \& Rozas 2009), which in turn used the Nei-Gojobori (NG) method(Li et al. 1985; Nei \& Gojobori 1986).

\section{Investigation on Cis-elements in the Promoter Region}

The 2500 bp genomic sequences upstream at the transcription start site (ATG) were extracted from Pyrus bretschneideri genomic database, which was used to analyze putative cis-elements in the promoter regions of the PEBP genes with PlantCARE (http://bioinformatics.psb.ugent.be/webtools/plantcare/html/).

\section{Plant Materials, Growth Conditions and Treatment}

The seven-year-old pear trees (Pyrus bretschneideri, cv. Xueqing) were used as the trail samples that were grown in a natural environment in Hebei Agricultural University $\left(\mathrm{N} 38^{\circ}, \mathrm{E} 115^{\circ}\right)$.

To clarify the expression of $P E B P$ genes in pears during the flower bud differentiation, leaf and apical bud samples at different developmental stages were collected at 30, 45, 60, 75, 90 and 105 days after full blooming (DAB). Meanwhile, the day-length data was obtained from the internet (https://richurimo.51240.com/). The treatment of reduction day-length on the pear leaves started from $30 \mathrm{DAB}$ to $120 \mathrm{DAB}$ in the next year. A branch of each pear tree was covered with an opaque bag from 6:30 pm until night (Fig. S1). The leaf samples were collected at 45, 75 and $105 \mathrm{DAB}$ in this experiment.

In order to verify the circadian rhythm of the genes, the leaf samples in the natural environment were collected from 9:00am to the next 6:00am, once for every three hours at 45, 75 and 105DAB.

Arabidopsis (Columbia-0) was used in this study. To generate PbFT overexpression transgenic plants, full-length cDNAs without the stop codon for PbFT were cloned into a PBI-121 vector in the sense orientation behind a cauliflower mosaic virus $35 \mathrm{~S}$ promoter. Phenotypes of the 
118 transgenic lines were examined with T4 generation. Arabidopsis plants were cultured under long 119 days (LD, light/dark, $16 \mathrm{~h} / 8 \mathrm{~h}$ ) in an artificially controlled growth room at $23^{\circ} \mathrm{C}$.

120 All samples that were needed to further analyze were harvested and quickly frozen in liquid

121

122

123

124

125

126

127

128

129

130

131

132

133

134

135

136

137

138

139

140

141

142

143

144

145

146

147

148

149

150

151

152

153

154

155

156

157

158 nitrogen and then stored at $-80^{\circ} \mathrm{C}$ for further experiments. At least three independent replications were carried out for all assaying samples.

\section{Meristem microscopy}

Apical buds of current shoots harvest at 60DAB, 75DAB and 90DAB were fixed quickly in FAA solution (70\% ethanol: formalin: acetic acid $=90: 5: 5, \mathrm{v} / \mathrm{v}$ ) and dehydrated in a graded ethanol series ( $1 \mathrm{~h}$ in each of $70 \%, 83 \%, 95 \%, 100 \%$ ethanol and $100 \%$ ethanol). Then, samples were soaked in mixtures of ethanol and xylene $(1: 1 \mathrm{v} / \mathrm{v}) 1 \mathrm{~h}$ and twice in absolute xylene for $1 \mathrm{~h}$. Subsequently, Samples were infiltrated at $37^{\circ} \mathrm{C}$ with mixtures of xylene and steedman's wax made up in the proportions of $1: 1(\mathrm{v} / \mathrm{v})$ overnight and at $45^{\circ} \mathrm{C} 12 \mathrm{~h}$, followed by two changes of pure wax ( $2 \mathrm{~h}$ each). Specimens were transferred to kraft paper molds filled in fresh wax at $50^{\circ} \mathrm{C}$, then cooling to room temperature. Samples were sectioned into $8 \mu \mathrm{m}$ slice and dewaxed before sealing. The slices were observed under a microscope and photographed.

\section{RNA Extraction, cDNA Synthesis and Quantitative Real-time PCR}

Total RNA was extracted according to the manufacturer instructions of E.Z.N.A. plant RNA extraction kit (Omega Bio-tek, Georgia, USA) including the gDNase. The first strand cDNA was prepared using FastQuant RT Kit (Tiangen, Beijing, China) according to the manufacturer's instructions together with gDNase.

Leaf samples harvested at $30 \mathrm{DAB}, 45 \mathrm{DAB}, 60 \mathrm{DAB}, 75 \mathrm{DAB}$ and $90 \mathrm{DAB}$ were using for transcriptome sequencing. Three independent replications were carried out for all assaying samples (Since samples harvested at 30DAB and 90DAB have been confirmed as not critical samples in our research (unsubmitted), both were not set biological replicates for transcriptome sequencing). The subsequent library construction and RNA-sequencing (RNA-seq) were completed by the Shanghai Majorbio Bio-pharm Technology Co., Ltd (Shanghai, China). The data was analyzed on the free online platform of Majorbio Cloud Platform (www.majorbio.com). The RNA-Seq data from this article can be found in the Sequence Read Archive (SRA) under accession number SRR10997902- SRR10997912.

The quantitative real-time PCR (qRT-PCR) reactions were executed using the TransStart ${ }^{\circledR}$ Top Green qPCR SuperMix (TransGen Biotech, Beijing, China). The qRT-PCR was performed using Eppendorf Mastercycler ${ }^{\circledR}$ ep realplex (Eppendorf, Germany). The thermal cycling conditions were $30 \mathrm{sec}$ at $94^{\circ} \mathrm{C} ; 44 \mathrm{cycles}$ for $5 \mathrm{sec}$ at $94^{\circ} \mathrm{C}, 15 \mathrm{sec}$ at $56^{\circ} \mathrm{C}$ and $10 \mathrm{sec}$ at $72^{\circ} \mathrm{C}$, and then performed the melting curve and cooling program. The data was calculated using the $2^{-\Delta \Delta \mathrm{Ct}}$ (Shi et al. 2019) method and the PbActin gene of Pyrus bretschneideri was used as an internal control to normalize the expression levels of the target genes. Specific primers of the qRT-PCR were presented in Table S1. Three biological replications were used for the experiment.

\section{Statistical Analysis}

Statistical Product and Service Solutions v. 17.0 (SPSS, Chicago, IL, USA) was used to analyze the experimental data. T-test and ANOVA Tukey's multiple comparison tests were used to exam significant differences $(\mathrm{p}<0.05)$. 
159

160

161

162

163

164

165

166

167

168

169

170

171

172

173

174

175

176

177

178

179

180

181

182

183

184

185

186

187

188

189

190

191

192

193

194

195

196

197

198

199

\section{Results}

\section{Genome-wide Identification of PEBP Genes}

Based on a genome-wide analysis, 24 PEBP genes were identified in pears, of which 10 PEBP genes were from Pyrus bretschneideri genome, 5 PEBP genes were from Pyrus communis genome and 9 PEBP genes were from the Pyrus betuleafolia genome. The detailed information of $P E B P$ genes is given in Table 1. The PEBP proteins varied more or less with respect to characters, ranging from 172 amino acids (aa) to 192 aa in length, from 18872.85 Da to 21386.94 Da in molecular weight, and a pI from 6.5 to 9.2 .

Mapping PEBP genes to the Pyrus bretschneideri genome indicated that 10 PEBP genes were unevenly distributed on eight of the seventeen chromosomes, with six genes on $\mathrm{Chr} 2, \mathrm{Chr} 3$, Chr5, Chr7, Chr11 and Chr12, as well as two on Chr6 or Chr14. Mapping PEBP genes to Pyrus communis genome indicated that 5 PEBP genes were unevenly distributed on five of the seventeen chromosomes, i.e. Chr1, Chr3, Chr4, Chr7, and Chr12. Mapping PEBP genes to Pyrus betuleafolia genome indicated that 9 PEBP genes were unevenly distributed on seven of the seventeen chromosomes, with five genes on Chr1, Chr4, Chr6, Chr7, and Chr11, as well as two on Chr12 or Chr14(Fig. 1).

\section{Phylogenetic Analysis of the $P E B P$ Family}

According to the phylogenetic tree, 24 PEBP genes of pears were clustered into three groups (TFL1-like, FT-like and MFT-like), and each group contained one Arabidopsis PEBP gene at least (Fig. 2A). The genes, including gene14557, gene20820, gene31806, gene23124, genel6540, gene20297, pycom03g09760, pycom01g20860, pycom07g24410, GWHPAAYT007785, GWHPAAYT010058, GWHPAAYT016784, GWHPAAYT001914 and GWHPAAYT050130, were clustered to TFL1-like group together with ATC, BFT and TFL-1. Among them, gene23124, gene16540, GWHPAAYT010058 and GWHPAAYT016784 were TFL1 homologues. Additionally, these genes, including gene 12374 , pycom12g24180, pycom04g21920, GWHPAAYT012924 and GWHPAAYT040350, were clustered to FT-like group together with TSF and FT. And those genes, including gene11252, gene7939, gene4010, GWHPAAYT019304 and GWHPAAYT047043, were clustered to MFT-like group together with AtMFT.

\section{Gene Structure and Conservative Motifs Analysis}

Gene structure analysis showed that all PEBP genes from pears contained four exons and three introns. The sizes of the second exon and the third exon were exactly $62 \mathrm{bp}$ and $41 \mathrm{bp}$ (Fig. 2B). Nine motifs (Fig. S2-S10) in pear PEBPs were detected and all pear PEBP proteins contained motifs 1 5. Motif 5 contained D-P-D-X-P and G-X-H-R residues, which were conserved residues to the $P E B P$ family of all plants. However, only $M F T$-like group proteins contained motifs $6 \sim 9$ (Fig. 2C).

\section{Selective Pressure Analysis}

The gene pairs were obtained by synteny analysis. The ratio of non-synonymous substitutions (Ka)/synonymous substitutions (Ks) was evaluated to detect the modes of selection of PEBP genes. Generally, $\mathrm{Ka} / \mathrm{Ks}>1$ indicated a positive selection with an accelerated evolution; $\mathrm{Ka} / \mathrm{Ks}$ $<1$ indicated functional constraints with a negative selection; and $\mathrm{Ka} / \mathrm{Ks}=1$ signified a neutral

Peer) reviewing PDF | (2020:01:44784:2:0:NEW 26 Feb 2020) 
200

201

202

203

204

205

206

207

208

209

210

211

212

213

214

215

216

217

218

219

220

221

222

223

224

225

226

227

228

229

230

231

232

233

234

235

236

237

238

239

240

241

selection. The results showed that the $\mathrm{Ka} / \mathrm{Ks}$ ratios of 13 duplicated $P E B P$ gene pairs ranged from 0.11 to 0.85 with an average of 0.27 (Table 2). Additionally, the majority of the ratios were less than 0.39 , suggesting that the duplicated $P E B P$ genes of pears had mainly undergone strong purifying selection, with the slowly evolving at the protein level of this gene family.

\section{Cis-elements in the PEBP Genes Promoters of Pyrus bretschneideri}

We analyzed 2500 bp genomic sequences upstream at the transcription start site (ATG) of Pyrus bretschneideri PEBP genes. About 50 putative cis-elements were identified in each promoter of $P E B P$ genes that were classified into three groups, including hormone responses, plant growth and development and biotic/abiotic stress responses (Figure 3). There were numerous lightresponsive elements on these promoters, such as AE-box, Box 4, G-box, I-box, and GT1-motif. The promoter of gene 12374 possessed three Auxin-responsive elements and one GA-responsive element, and the promoter of gene 23124 had one Auxin-responsive element, which was not found in the promoters of other Pyrus bretschneideri PEBP genes.

\section{Expression of $P E B P$ Genes during the Flower Bud Differentiation}

Expression of pear PEBP genes obtained from the RNA-seq data of 'Xueqing' leaves at different stages was analyzed (Table S2). It was found that just one gene (gene12374) was expressed in all five development stages, and was relatively highly expressed in leaves (Fig. 4A). The other genes had low or no expression. Phylogenetic analysis revealed that the closest relationship between gene12374 and Arabidopsis PEBP gene was FT, so gene12374 was re-named PbFT. $P b F T$ expression patterns with the qRT-PCR experiment were shown in Figure 4B. The expression trends of $P b F T$ were similar to RNA-seq data. The highest expression of $P b F T$ was consistently observed at 75DAB. Then, the day-length of a period from 30DAB to $120 \mathrm{DAB}$ was recorded (Fig. 4C). According to the microscopic observation of current shoot apical meristems, it was not found abnormal at $60 \mathrm{DAB}$. This period was maybe in the flower bud physiological differentiation stage. Whereas, it was found that the flower bud morphological differentiation stage had already entered at 75DAB (Fig. 4D-F). Meanwhile, the expressions of PbFT was also the highest in apical buds (Fig. 5A). Those findings suggested that the period from 60DAB to $75 \mathrm{DAB}$ was critical for the flower bud differentiation, and the expression of $P b F T$ was increasing strongly during this period. Additionally, the expressions of two TFL1 homologues (gene23124 and gene16540) were higher before 60DAB in apical buds. The lowest expressions of gene23124 and gene16540 occurred at 75DAB. Subsequently, the expression of gene23124 remained at a lower level, but the expression of gene 16540 increased gradually (Fig. 5B and C). Interestingly, the time of the highest expression of $P b F T$ was found around the day with the longest daylength. Subsequently, the treatment of day-length reduction on the pear leaves showed that if the day-length was reduced, the expression of PbFT also decreased (Fig. 6). The expression of $P b F T$ under the natural condition was up to twice as much as under the treatment of day-length reduction at 75DAB.

On the other hand, the expression of PbFT was at a high level during the day, but it dropped suddenly as night fell and remained at a lower level until dawn (Fig. 7).

\section{Overexpression of $\mathrm{PbFT}$ in Relation to Acceleration of Flowering Time}

To investigate the role of $P b F T$ in flower bud induction regulation, $P b F T$ was overexpressed under the control of the $35 \mathrm{~S}$ promoter in the wild-type Arabidopsis (Col-0). A total of 9 
242 independent lines of overexpressing PbFT (PbFT-OE) plants that showed obviously enhanced 243 expression of $P b F T$ were obtained. Two independent lines were randomly selected for further 244 analysis. The transgenic lines were confirmed by PCR with the 35S forward primer (35S-F) and 245 PbFT-specific reverse sequencing primer (PbFT-R) (Table S1). The days from germination to 246 flowering was 32.11 for WT plants, but 24.33 and 25.33 with PbFT-OE3 and PbFT-OE9 lines. 247 The corresponding total rosette leaf number was 18.29, 8.57 and 10.17 in WT, PbFT-OE3 and 248 PbFT-OE9 lines. The days from germination to flowering were significantly accelerated and the 249 total rosette leaf number decreased significantly in the PbFT-OE lines compared with the WT 250 plants, but two overexpression lines showed no significant difference in the relative parameters 251 (Fig. 8). The diameters of the transgenic lines PbFT-OE3 and PbFT-OE9 main stem were 0.76 252 and $0.82 \mathrm{~mm}$, which were significantly larger than $0.5 \mathrm{~mm}$ of WT. In the absence of human 253 interference, the transgenic plants were lodging, as opposing to the wild-type upright phenotype 254 (Fig. 8).

\section{Discussion}

\section{Conservation and Evolution of $P E B P$ genes in pears}

257 So far, the genome sequencing of 'DangshanSuli' (Pyrus bretschneideri), 'Bartlett' (Pyrus 258 communis) and 'Shanxi Duli' (Pyrus betuleafolia) has been completed. These existing 259 information resources can be used to analyze the evolution and gene function of the PEBP 260 family from the perspective of bioinformatics. The PEBPs were a kind of evolutionarily 261 conserved proteins. As the PEBP genes of other species like Arabidopsis and apple (Malus 262 domestica), the $P E B P$ genes of pears consisted of four exons and three introns, and meanwhile 263 the second exon contained 62 bases and the third exon contained 41 bases. In addition, the $\mathrm{Ka} / \mathrm{Ks}$ 264 of $P E B P$ gene pairs obtained from collinearity analysis in different genera were far less than 1. 265 This result indicated that the $P E B P$ genes of pears have undergone purifying selections, which 266 also confirmed that the $P E B P$ family was quite conservative. However, the number of $P E B P$ 267 genes and their distribution on chromosomes of Pyrus bretschneideri, Pyrus communis and 268 Pyrus betuleafolia were different in this study. Ten and nine genes from Pyrus bretschneideri 269 and Pyrus betuleafolia were identified, while only five genes were identified from Pyrus 270 betuleafolia. This result suggested that different degrees of gene loss had occurred during the 271 evolution of Pyrus, especially in the Pyrus communis genome, at least in the PEBP family of 272 'Bartlett' pear. Interestingly, among the materials used for genome sequencing, 'DangshanSuli' 273 (Pyrus bretschneideri) and 'Shanxi Duli' (Pyrus betuleafolia) belonged to Asian pears, and 274 'Bartlett' (Pyrus communis) was a European pear. This situation might be due to the independent 275 domestication processes for either Asian or European pears(Wu et al. 2018).

276 In addition, more attention should be paid to the genes in the FT-like group. FT and TWIN 277 SISTER OF FT (TSF) had redundant functions in promoting flowering (Jin et al. 2015; 278 Yamaguchi et al. 2005), and they had five ortholog genes in pears. There were two genes 279 belonging to both 'Bartlett' and 'Shanxi Duli', but just one, $P b F T$, was identified in 280 'DangshanSuli' genome. Since PbFT was so closely related to the AtFT and AtTSF, we 281 speculated that it may also be similar in functions.

\section{Expression and Function of PbFT}

283 FT and TFL1 played important roles in flowering induction(Gao et al. 2017; Putterill \& 284 Varkonyi-Gasic 2016). The expressions of PbFT and TFL1 homologues (gene23124 and 
285

286

287

288

289

290

291

292

293

294

295

296

297

298

299

300

301

302

303

304

305

306

307

308

309

310

311

312

313

314

315

316

317

318

319

320

321

322

323

324

325

326

327

328

329

330

gene16540) in pear buds showed the opposite expression patterns before and in the critical period of the flower bud differentiation. However, it was found that only $P b F T$ among pear $P E B P$ genes was relatively highly expressed in leaves. It suggested that $P b F T$ played positive roles both in leaves and buds, but gene23124 and gene16540 played negative roles only in buds. In Arabidopsis, TFL1 expression was merely limited to shoots(Baumann et al. 2015). This was similar to our results. It suggested that gene23124 and gene16540 had the opposite function to $P b F T$. As a receptor of the plant, the leaves can directly sense the changes of the external environment, especially the time of day-length, so as to regulate the growth and development of the plant through corresponding signal transduction. FT protein is synthesized in the leaf vasculature and transported to the apical meristem of a shoot through the phloem(Corbesier et al. 2007; Lin et al. 2007; Tamaki et al. 2007; Zeevaart 2008). By analyzing the promoter, it was found that there were typical light-responsive elements, AE-box, Box 4, G-box and TCT-motif, on the PbFT promoter. This suggested that the expression of PbFT may be regulated by light. According to our transcriptome data, only the PbFT gene, among the PEBP genes of Pyrus bretschneideri, had a relatively high expression level in the leaves. It was found that the expression of $P b F T$ was increasing strongly during critical period, which was from the flower bud physiological differentiation stage to morphological differentiation stage, and the highest expression of PbFT was observed at 75DAB, when was about the longest daytime of a year. Shortening the day-length of some branches indicated that the expression of $P b F T$ was downregulated. This was a good indication that the expression of the PbFT gene was related to the flower bud induction and considerably regulated by the day-length in a year.

Many scholars have concluded that $F T$ is strongly expressed around dusk in LD in the incubator (Mouradov et al. 2002; Song et al. 2013). However, we found that PbFT gene remained at a high expression level during the day and at a lower level in the evening until dawn. PbFT expression showed an unusual circadian rhythm. As the temperature and light in the incubator are usually constant but greater changes exist in the natural environment, these may result in a different pathway of regulation mechanism with $P b F T$.

At least six signal pathways (photoperiod, autonomous, age, gibberellin, vernalization, ambient temperature) that regulate the flowering process have been demonstrated(Blumel et al. 2015; Kim et al. 2009; Mutasa-Gottgens \& Hedden 2009; Srikanth \& Schmid 2011; Wang 2014). As a mobile signal florigen gene, in several pathways, FT can initiate the floral induction in some species (Blumel et al. 2015; Cao et al. 2015; Corbesier et al. 2007; Liu et al. 2013; Oda et al. 2012; Pin \& Nilsson 2012; Tamaki et al. 2007). The research on FT genes has been progressed in many horticultural plants (Wilkie et al. 2008). However, the role of the PbFT during flower bud differentiation remains unclear in pears. In this study, it was found the expression of $P b F T$ increased during the period of floral initiation. Moreover, the overexpression of $P b F T$ dramatically accelerated the starting time for flowering Arabidopsis. This implies that the PbFT might play a role in promoting flower bud differentiation in pears.

Additionally, homologs of FT genes in crops have pleiotropic functions that can mediate numerous developmental processes, such as growth, plant architecture control, fruit set and tuber formation(Pin \& Nilsson 2012). AcFT1 promotes bulb formation, while AcFT4 inhibits bulb production in onions(Lee et al. 2013). Tuberization is regulated by the FT homolog StSP6A through modulation of source-sink in potatoes (Abelenda et al. 2019; Lehretz et al. 2019). Our transgenic plants were thinner than WT, which was caused by overexpression of $P b F T$. This finding suggests that $P b F T$ has a role in not only promoting flower bud differentiation, but also regulating the balance between vegetative and reproductive growth.

Peer] reviewing PDF | (2020:01:44784:2:0:NEW 26 Feb 2020) 


\section{Conclusions}

333

334

335

336

337

338

339

340

341

\section{2}

343

344

345

346

347

348

349

350

351

352

353

354

355

356

357

358

359

360

361

362

363

364

365

366

367

368

369

370

371

372

373

374

375

We identified 10, 5 and 9 PEBP genes from genomes of Pyrus bretschneideri, Pyrus communis and Pyrus betuleafolia, respectively. The PEBPs is a kind of evolutionarily conserved proteins. However, the number of PEBP genes and their distribution on chromosomes of Pyrus bretschneideri, Pyrus communis and Pyrus betuleafolia are different. This situation may be due to the independent domestication processes for either Asian or European pears. Moreover, we determined that $P b F T$, which can be regulated by day-length and circadian clock, possessed the function of promoting flower bud differentiation in pears. Additionally, PbFT may play a role in regulating the balance between vegetative and reproductive growth.

\section{Acknowledgements}

We sincerely thank Professor Jiafu Jiang (Nanjing Agricultural University) for providing the Arabidopsis seeds.

\section{References}

\section{Abelenda JA, Bergonzi S, Oortwijn M, Sonnewald S, Du MR, Visser RGF, Sonnewald U, and Bachem CWB. 2019. Source-Sink Regulation Is Mediated by Interaction of an FT Homolog with a SWEET Protein in Potato. Current Biology 29:1178-+. 10.1016/j.cub.2019.02.018}

Baumann K, Venail J, Berbel A, Domenech MJ, Money T, Conti L, Hanzawa Y, Madueno F, and Bradley D. 2015. Changing the spatial pattern of TFL1 expression reveals its key role in the shoot meristem in controlling Arabidopsis flowering architecture. J Exp Bot 66:47694780. 10.1093/jxb/erv247

Blumel M, Dally N, and Jung C. 2015. Flowering time regulation in crops-what did we learn from Arabidopsis? Curr Opin Biotechnol 32:121-129. 10.1016/j.copbio.2014.11.023

Cao K, Cui L, Zhou X, Ye L, Zou Z, and Deng S. 2015. Four Tomato FLOWERING LOCUS TLike Proteins Act Antagonistically to Regulate Floral Initiation. Front Plant Sci 6:1213. 10.3389/fpls.2015.01213

Chautard H, Jacquet M, Schoentgen F, Bureaud N, and Benedetti H. 2004a. Tfs $1 p$, a Member of the PEBP Family, Inhibits the Ira2p but Not the Ira1p Ras GTPase-Activating Protein in Saccharomyces cerevisiae. Eukaryotic Cell 3:459-470.

Chautard H, Jacquet M, Schoentgen F, Bureaud N, and Bénédetti H. 2004b. Tfs 1p, a member of the PEBP family, inhibits the Ira2p but not the Ira1p Ras GTPase-activating protein in Saccharomyces cerevisiae. Eukaryotic Cell 3:459.

Chen C, Xia R, Chen H, and He Y. 2018. TBtools, a Toolkit for Biologists integrating various HTS-data handling tools with a user-friendly interface. biorxiv:289660. 10.1101/289660

Corbesier L, Vincent C, Jang S, Fornara F, Fan Q, Searle I, Giakountis A, Farrona S, Gissot L, Turnbull C, and Coupland G. 2007. FT protein movement contributes to long-distance signaling in floral induction of Arabidopsis. Science 316:1030-1033.

10.1126/science. 1141752

Peer) reviewing PDF | (2020:01:44784:2:0:NEW 26 Feb 2020) 
376

377

378

379

380

381

382

383

384

385

386

387

388

389

390

391

392

393

394

395

396

397

398

399

400

401

402

403

404

405

406

407

408

409

410

411

412

413

414

415

416

417

418

419

420

421

422

423

424

425

Danilevskaya ON, Meng X, Hou Z, Ananiev EV, and Simmons CR. 2008. A genomic and expression compendium of the expanded PEBP gene family from maize. Plant Physiol 146:250-264. 10.1104/pp.107.109538

Finn RD, Coggill P, Eberhardt RY, Eddy SR, Mistry J, Mitchell AL, Potter SC, Punta M, Qureshi M, Sangrador-Vegas A, Salazar GA, Tate J, and Bateman A. 2016. The Pfam protein families database: towards a more sustainable future. Nucleic Acids Res 44:D279-285. 10.1093/nar/gkv1344

Gao J, Huang BH, Wan YT, Chang J, Li JQ, and Liao PC. 2017. Functional divergence and intron variability during evolution of angiosperm TERMINAL FLOWER1 (TFL1) genes. Sci Rep 7:14830. 10.1038/s41598-017-13645-0

Hu B, Jin J, Guo AY, Zhang H, Luo J, and Gao G. 2014. GSDS 2.0: an upgraded gene feature visualization server. Bioinformatics 31:1296.

Igasaki T, Watanabe Y, Nishiguchi M, and Kotoda N. 2008. The FLOWERING LOCUS T/TERMINAL FLOWER 1 family in Lombardy poplar. Plant Cell Physiol 49:291-300. 10.1093/pcp/pcn010

Jin S, Jung HS, Chung KS, Lee JH, and Ahn JH. 2015. FLOWERING LOCUS T has higher protein mobility than TWIN SISTER OF FT. J Exp Bot 66:6109-6117. 10.1093/jxb/erv326

Johnson LS, Eddy SR, and Portugaly E. 2010. Hidden Markov model speed heuristic and iterative HMM search procedure. BMC Bioinformatics 11:431. 10.1186/1471-2105-11431

Karlgren A, Gyllenstrand N, Kallman T, Sundstrom JF, Moore D, Lascoux M, and Lagercrantz U. 2011. Evolution of the PEBP gene family in plants: functional diversification in seed plant evolution. Plant Physiol 156:1967-1977. 10.1104/pp.111.176206

Kim DH, Doyle MR, Sung S, and Amasino RM. 2009. Vernalization: winter and the timing of flowering in plants. Annu Rev Cell Dev Biol 25:277-299. 10.1146/annurev.cellbio.042308.113411

Lee R, Baldwin S, Kenel F, McCallum J, and Macknight R. 2013. FLOWERING LOCUS T genes control onion bulb formation and flowering. Nat Commun 4:2884. 10.1038/ncomms3884

Leeggangers $\mathrm{H}$, Rosilio-Brami T, Bigas-Nadal J, Rubin N, van Dijk ADJ, Nunez de Caceres Gonzalez FF, Saadon-Shitrit S, Nijveen H, Hilhorst HWM, Immink RGH, and Zaccai M. 2018. Tulipa gesneriana and Lilium longiflorum PEBP Genes and Their Putative Roles in Flowering Time Control. Plant Cell Physiol 59:90-106. 10.1093/pcp/pcx164

Lehretz GG, Sonnewald S, Hornyik C, Corral JM, and Sonnewald U. 2019. Post-transcriptional Regulation of FLOWERING LOCUS T Modulates Heat-Dependent Source-Sink Development in Potato. Curr Biol 29:1614-1624 e1613. 10.1016/j.cub.2019.04.027

Li C, Zhang Y, Liu H, and Huang X. 2015. Identification of PEBP Gene Family in Gossypium arboreum and Gossypium raimondii and Expression Analysis of the Gene Family in Gossypium hirsutum. ACTA AGRONOMICA SINICA.

$\mathrm{Li} \mathrm{WH}, \mathrm{Wu} \mathrm{Cl}$, and Luo CC. 1985. A new method for estimating synonymous and nonsynonymous rates of nucleotide substitution considering the relative likelihood of nucleotide and codon changes. Mol Biol Evol 2:150-174.

10.1093/oxfordjournals.molbev.a040343

Librado P, and Rozas J. 2009. DnaSP v5: a software for comprehensive analysis of DNA polymorphism data. Bioinformatics 25:1451-1452. 10.1093/bioinformatics/btp187

Lin MK, Belanger H, Lee YJ, Varkonyi-Gasic E, Taoka K, Miura E, Xoconostle-Cazares B, Gendler K, Jorgensen RA, Phinney B, Lough TJ, and Lucas WJ. 2007. FLOWERING LOCUS T protein may act as the long-distance florigenic signal in the cucurbits. Plant Cell 19:1488-1506. 10.1105/tpc.107.051920

Liu L, Zhu Y, Shen L, and Yu H. 2013. Emerging insights into florigen transport. Curr Opin Plant Biol 16:607-613. 10.1016/j.pbi.2013.06.001

Peer) reviewing PDF | (2020:01:44784:2:0:NEW 26 Feb 2020) 
426

427

428

429

430

431

432

433

434

435

436

437

438

439

440

441

442

443

444

445

446

447

448

449

450

451

452

453

454

455

456

457

458

459

460

461

462

463

464

465

466

467

468

469

470

471

472

473

474

Mouradov A, Cremer F, and Coupland G. 2002. Control of flowering time: interacting pathways as a basis for diversity. Plant Cell 14 Suppl:S111-S130. 10.1105/tpc.001362

Mutasa-Gottgens E, and Hedden P. 2009. Gibberellin as a factor in floral regulatory networks. $J$ Exp Bot 60:1979-1989. 10.1093/jxb/erp040

Nei M, and Gojobori T. 1986. Simple methods for estimating the numbers of synonymous and nonsynonymous nucleotide substitutions. Molecular Biology \& Evolution 3:418-426.

Oda A, Narumi T, Li T, Kando T, Higuchi Y, Sumitomo K, Fukai S, and Hisamatsu T. 2012. CsFTL3, a chrysanthemum FLOWERING LOCUS T-like gene, is a key regulator of photoperiodic flowering in chrysanthemums. J Exp Bot 63:1461-1477. 10.1093/jxb/err387

Peng FY, Hu Z, and Yang RC. 2015. Genome-Wide Comparative Analysis of Flowering-Related Genes in Arabidopsis, Wheat, and Barley. Int J Plant Genomics 2015:874361. $10.1155 / 2015 / 874361$

Pin PA, and Nilsson O. 2012. The multifaceted roles of FLOWERING LOCUS T in plant development. Plant Cell Environ 35:1742-1755. 10.1111/j.1365-3040.2012.02558.x

Putterill J, and Varkonyi-Gasic E. 2016. FT and florigen long-distance flowering control in plants. Curr Opin Plant Biol 33:77-82. 10.1016/j.pbi.2016.06.008

Shi H, Zhang Y, and Chen L. 2019. Expression and Regulation of PpEIN3b during Fruit Ripening and Senescence via Integrating SA, Glucose, and ACC Signaling in Pear (Pyrus pyrifolia Nakai. Whangkeumbae). Genes 10. 10.3390/genes10060476

Song YH, Ito S, and Imaizumi T. 2013. Flowering time regulation: photoperiod- and temperature-sensing in leaves. Trends Plant Sci 18:575-583. 10.1016/j.tplants.2013.05.003

Srikanth A, and Schmid M. 2011. Regulation of flowering time: all roads lead to Rome. Cell Mol Life Sci 68:2013-2037. 10.1007/s00018-011-0673-y

Sun W, Shi J, Wu J, Zhang J, and Li N. 2018. A modified HLA-A*0201-restricted CTL epitope from human oncoprotein (hPEBP4) induces more efficient antitumor responses. Cellular \& Molecular Immunology 15.

Tamaki S, Matsuo S, Wong HL, Yokoi S, and Shimamoto K. 2007. Hd3a protein is a mobile flowering signal in rice. Science 316:1033-1036. 10.1126/science.1141753

Tamura K, Stecher G, Peterson D, Filipski A, and Kumar S. 2013. MEGA6: Molecular Evolutionary Genetics Analysis version 6.0. Mol Biol Evol 30:2725-2729. 10.1093/molbev/mst197

Voogd C, Brian LA, Wang T, Allan AC, and Varkonyi-Gasic E. 2017. Three FT and multiple CEN and BFT genes regulate maturity, flowering, and vegetative phenology in kiwifruit. $J$ Exp Bot 68:1539-1553. 10.1093/jxb/erx044

Wang JW. 2014. Regulation of flowering time by the miR156-mediated age pathway. $J$ Exp Bot 65:4723-4730. 10.1093/jxb/eru246

Wang M, Tan Y, Cai C, and Zhang B. 2018. Identification and expression analysis of phosphatidy ethanolamine-binding protein (PEBP) gene family in cotton. Genomics. 10.1016/j.ygeno.2018.09.009

Wilkie JD, Sedgley M, and Olesen T. 2008. Regulation of floral initiation in horticultural trees. $J$ Exp Bot 59:3215-3228. 10.1093/jxb/ern188

Wu J, Wang Y, Xu J, Korban SS, Fei Z, Tao S, Ming R, Tai S, Khan AM, Postman JD, Gu C, Yin H, Zheng D, Qi K, Li Y, Wang R, Deng CH, Kumar S, Chagne D, Li X, Wu J, Huang X, Zhang H, Xie Z, Li X, Zhang M, Li Y, Yue Z, Fang X, Li J, Li L, Jin C, Qin M, Zhang J, Wu X, Ke Y, Wang J, Yang H, and Zhang S. 2018. Diversification and independent domestication of Asian and European pears. Genome Biol 19:77. 10.1186/s13059-0181452-y 
475 Yamaguchi A, Kobayashi Y, Goto K, Abe M, and Araki T. 2005. TWIN SISTER OF FT (TSF)

476

477 acts as a floral pathway integrator redundantly with FT. Plant Cell Physiol 46:1175-1189. 10.1093/pcp/pci151

478

479

480

481

482

483

484

485

Yeung K, Seitz T, Li S, Janosch P, McFerran B, Kaiser C, Fee F, Katsanakis KD, Rose DW, Mischak H, Sedivy JM, and Kolch W. 1999. Suppression of Raf-1 kinase activity and MAP kinase signalling by RKIP. Nature 401:173-177. 10.1038/43686

Zeevaart JA. 2008. Leaf-produced floral signals. Curr Opin Plant Biol 11:541-547. 10.1016/j.pbi.2008.06.009

Zhang LF, Ran X, Zhang YW, Dai HY, and Zhang J. 2015. Analysis of the PEBP gene family in soybean (Glycine max). Journal of Plant Genetic Resources 16:151-157. 
Figure 1

Localization and synteny of the PEBP genes in pear genomes

(A) PEBP genes in Pyrus bretschneideri genome. (B) PEBP genes in Pyrus communis genome.

(C) PEBP genes in Pyrus betuleafolia genome. Chromosome number was indicated on the inner side of the inner circle. Gene pair with a syntenic relationship was joined by the line.
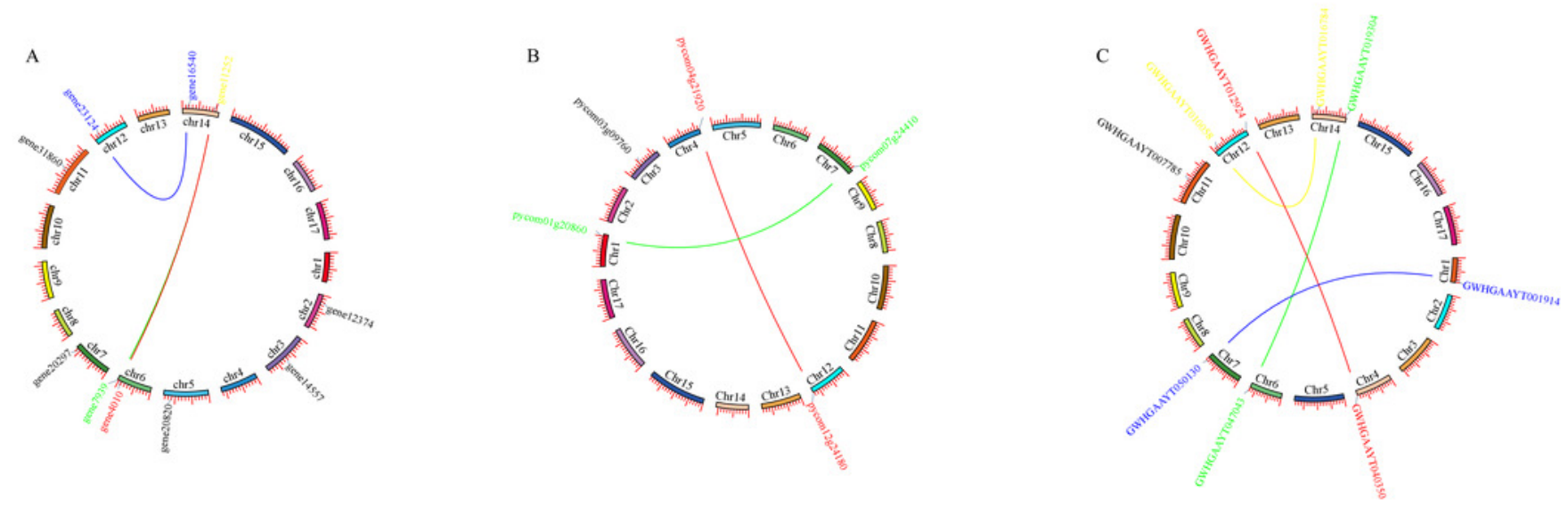
Figure 2

Phylogenetic relationships, gene structure and motifs in PEBP genes from pears.

(A) The phylogenetic tree was constructed based on the full-length sequences of pear PEBP proteins using MEGA 6.0 software. (B) Exon-intron structure of pear PEBP genes. Blue boxes indicated untranslated 5'- and 3'-regions; yellow boxes indicated exons; black lines indicated introns. (C) The motif composition of pear PEBP proteins. The motifs, numbers 1-9, were displayed in different colored boxes. The sequence information for each motif was provided in Figure S2-S10.

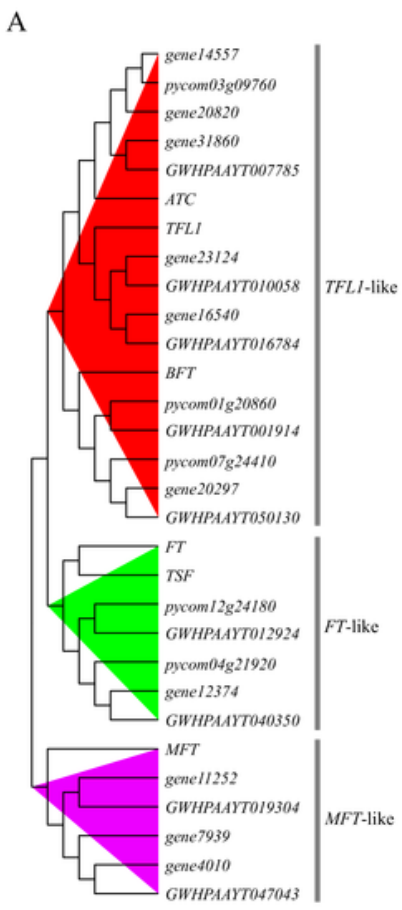

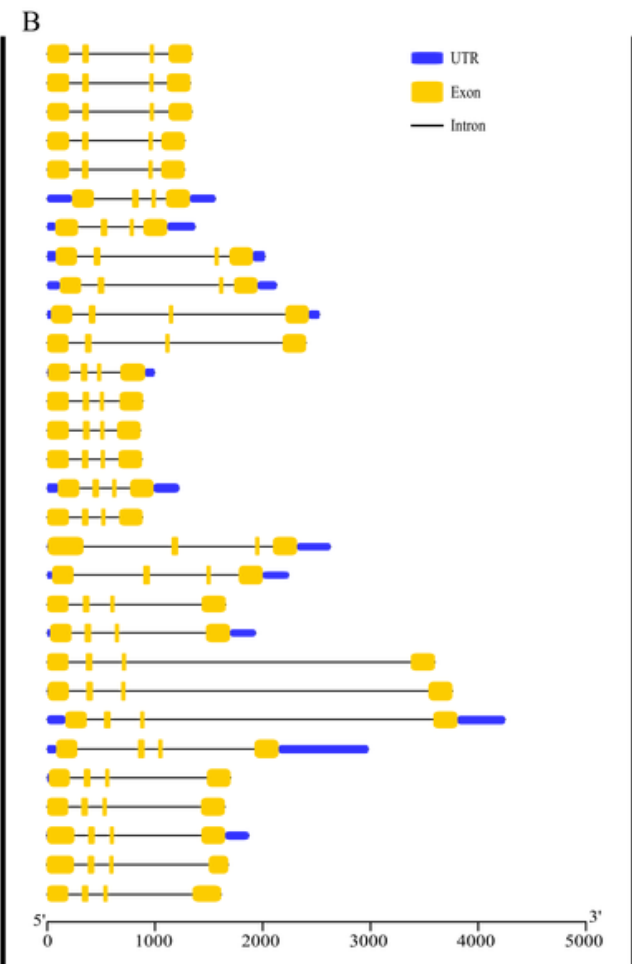

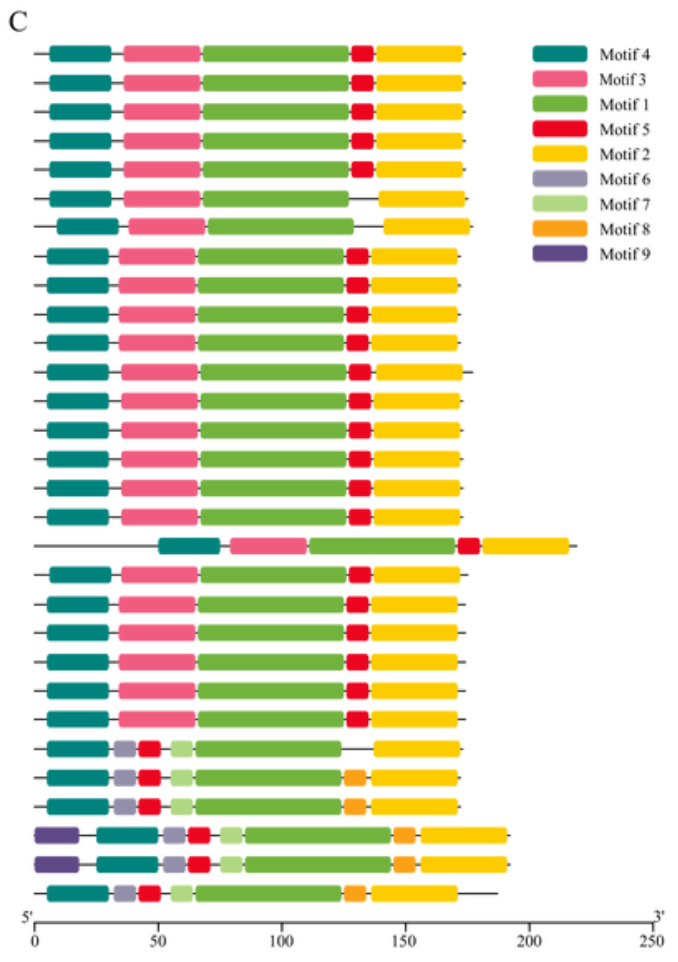


Figure 3

Investigation of cis-acting element numbers in the PEBP Genes Promoters of Pyrus bretschneideri.

The different colors and numbers of the grid indicated the numbers of different promoter elements in these PEBP genes.

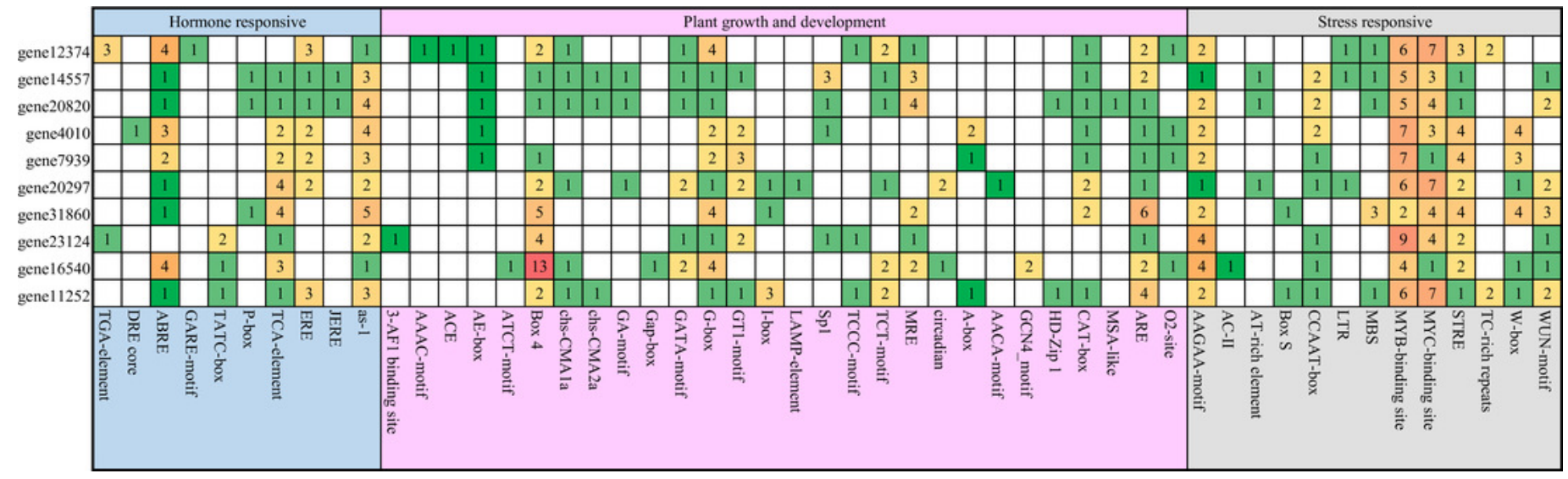




\section{Figure 4}

Expression profiles of PEBP genes in 'Xueqing' leaves during different stages.

(A) Heatmap of PEBP genes expression. (B) Relative expression of PbFT in 'Xueqing' leaves during different stages. (C) The day-length of a period from 30 DAB to 105DAB. Microscopy of current shoot apical meristems at (D)60DAB, (E)75DAB and (F)90DAB. Red arrows pointed to apical meristems. Scale bar, $100 \mu \mathrm{m}$.

A

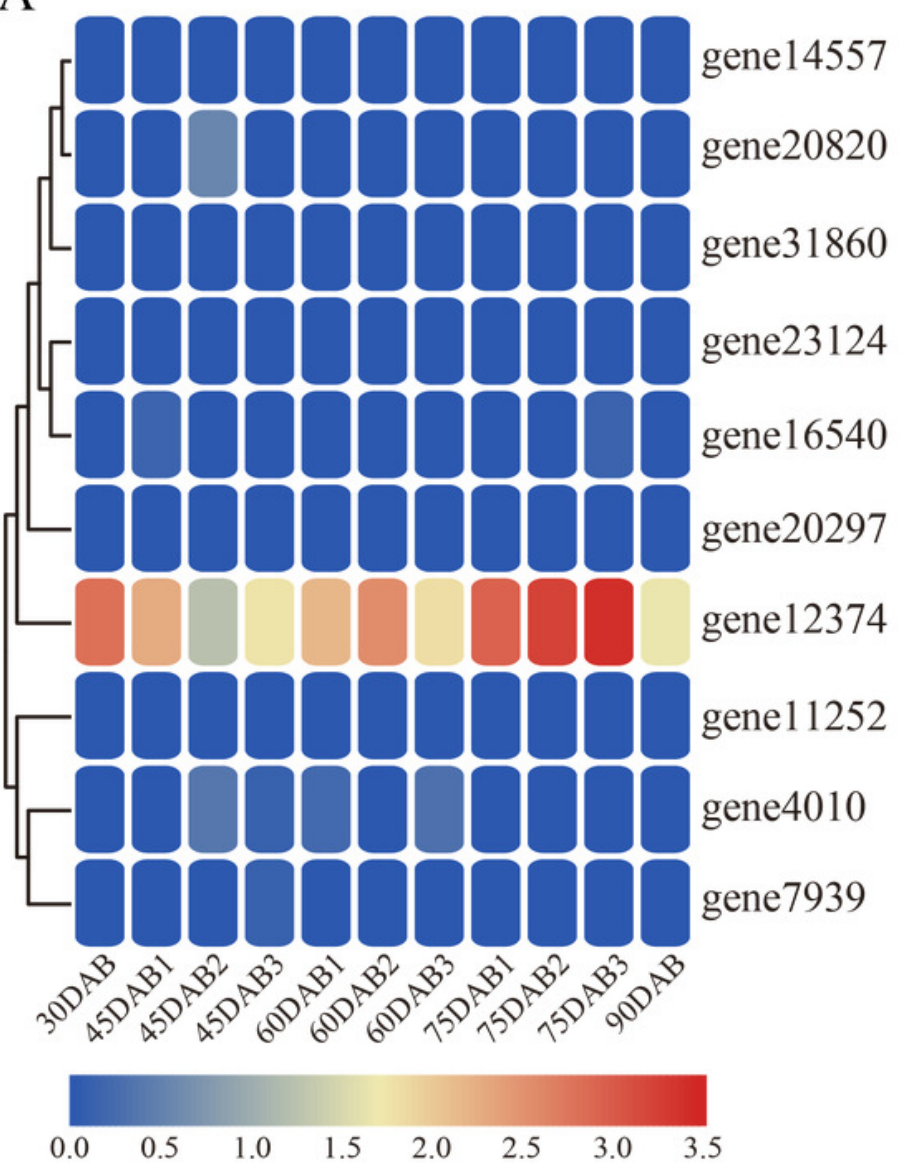

B

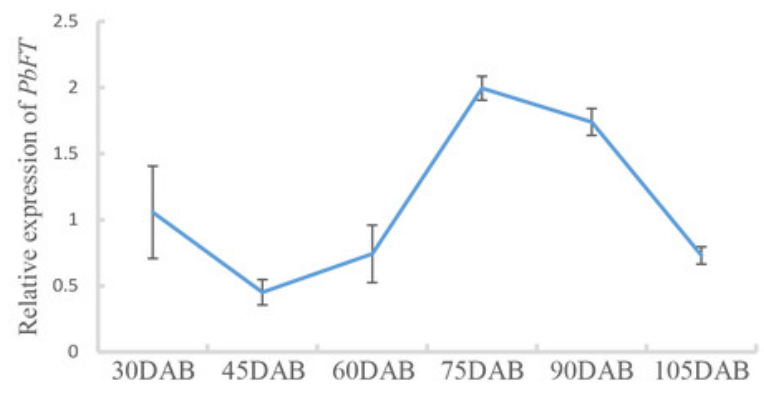

$\mathrm{C}$

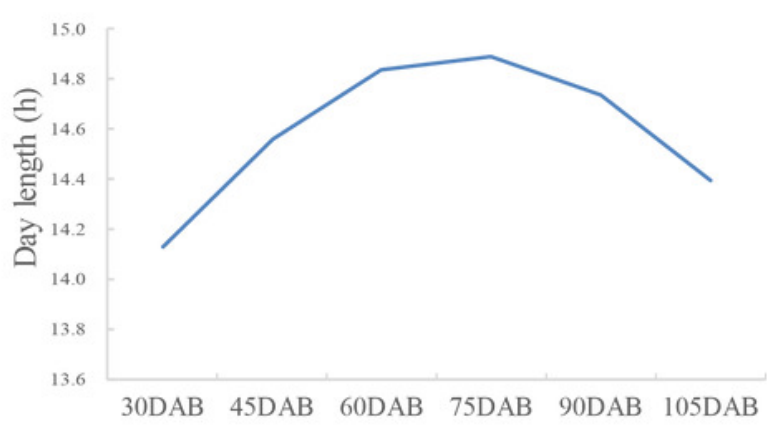

D

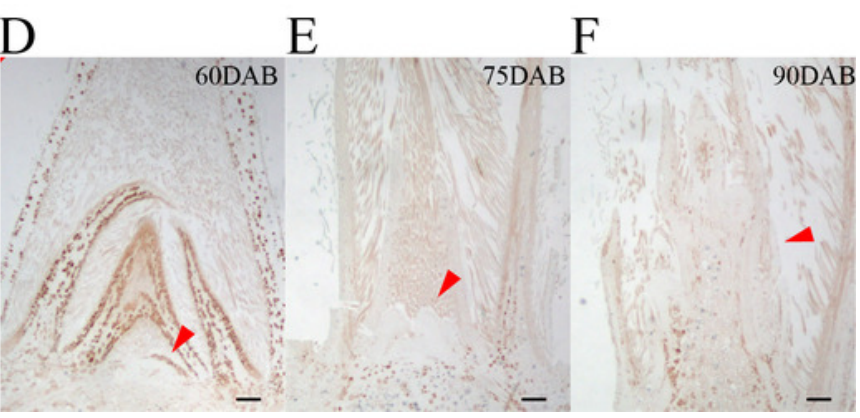


Figure 5

Relative expressions of PbFT and TFL1 homologues in 'Xueqing' buds during different stage

Relative expression of (A) PbFT, (B) gene23124 and (C) gene16540.
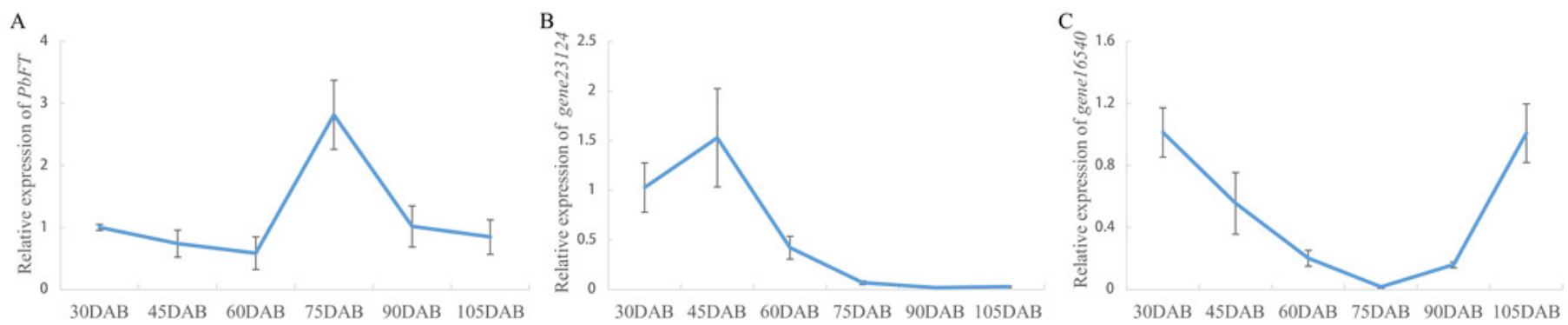
Figure 6

The relationship between relative expression of $P b F T$ and day-length.

(A) Compairon the day-length between the treatment and natural condition. (B) Comparison the relative expression of $P b F T$ between under the treatment and natural condition. Results were presented as mean $\pm S E$. $(n=3, * P<0.05)$
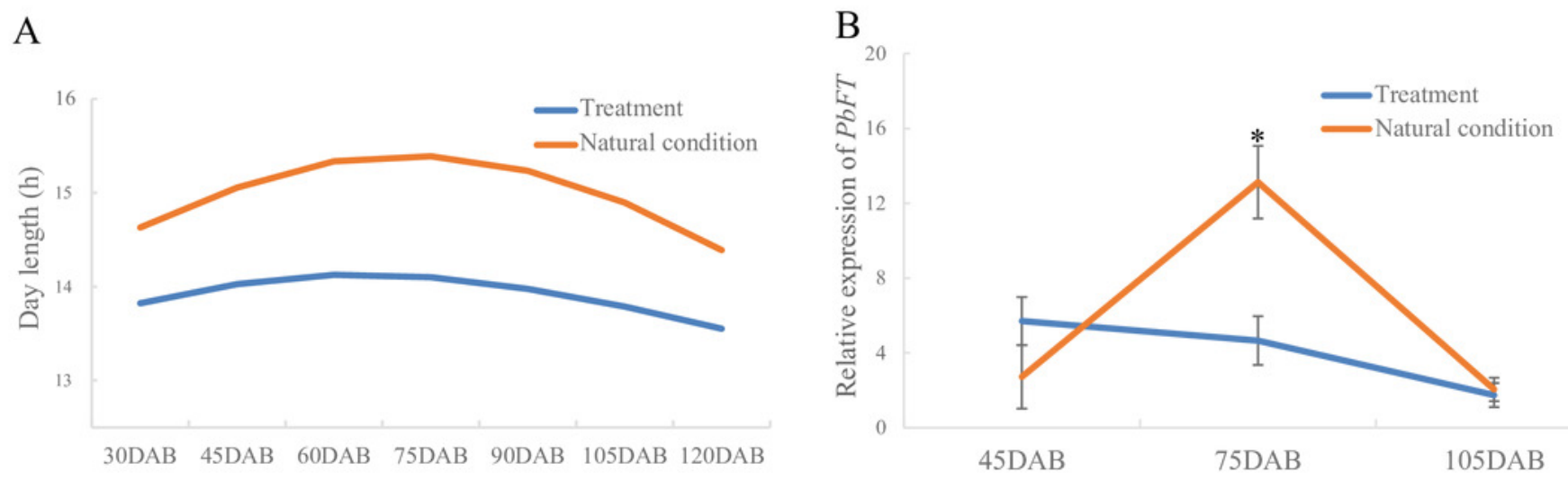
Figure 7

The circadian pattern of $P b F T$ expression.

Relative expression of PbFT in (A) 45DAB, (B) 75DAB and (C) 115DAB.
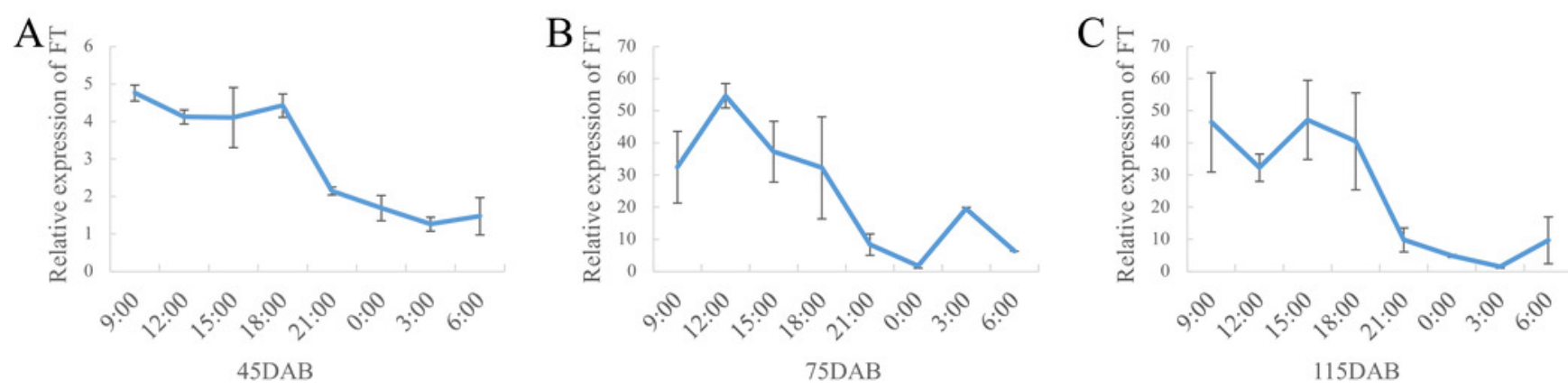


\section{Figure 8}

Flowering phenotype of 35S:PbFT transgenic Arabidopsis.

Representative images of transgenic Arabidopsis showing their flowering phenotype at $(A)$ $30 \mathrm{~d}$ and (B) $60 \mathrm{~d}$ after germination. Flowering phenotype of overexpressing PbFT transgenic Arabidopsis assessed by $(C)$ the expression of $P b F T$, (D)days from germination to flowering, and (E)rosette leaf number. (F) The diameters comparison of overexpressing PbFT transgenic Arabidopsis and WT. Results were presented as mean \pm SE. $(n \geq 3, *, p<0.05$, Students ttest).

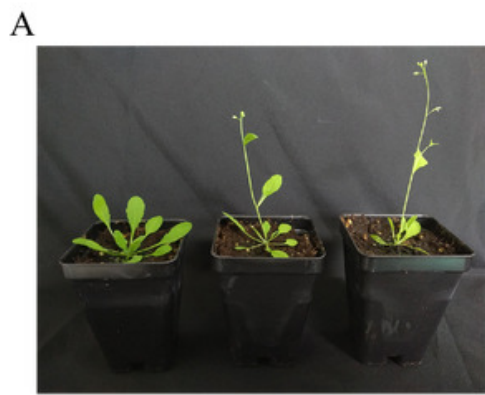

WT

PbFT-OE3 PbFT-OE9

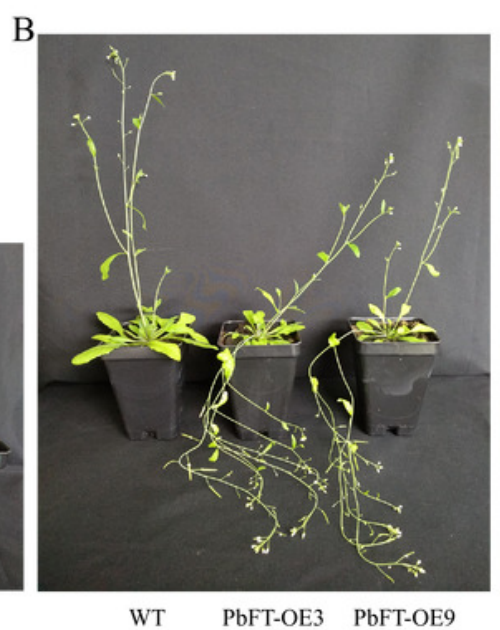

WT PbFT-OE3 PbFT-OE9
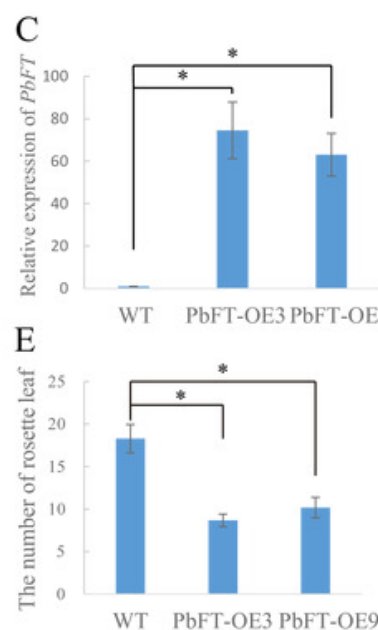

WT PbFT-OE3 PbFT-OE9

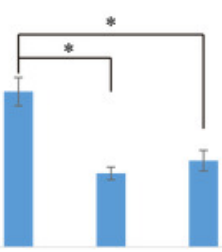

WT PbFT-OE3 PbFT-OE9
$\mathrm{D}$
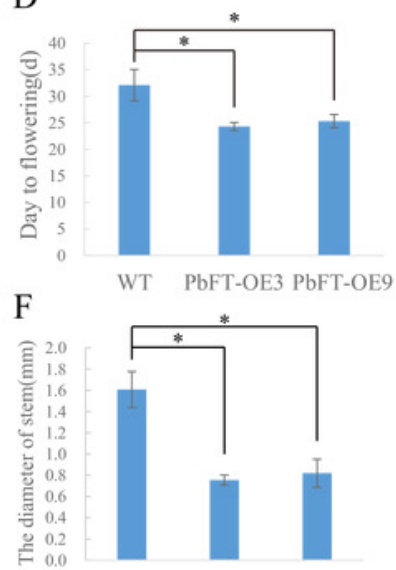

WT PbFT-OE3 PbFT-OE9 
Table $\mathbf{1}$ (on next page)

Characteristics of the PEBP genes identified in pears. 


\begin{tabular}{|c|c|c|c|c|c|c|c|}
\hline & $\begin{array}{l}\text { Accession } \\
\text { number }\end{array}$ & $\begin{array}{c}\text { Chromosome } \\
\text { location }\end{array}$ & $\begin{array}{c}\text { gene } \\
\text { length(bp) }\end{array}$ & $\begin{array}{c}\text { Exon } \\
\text { number }\end{array}$ & $\begin{array}{l}\text { Protein } \\
\text { length } \\
\text { (aa) }\end{array}$ & $\begin{array}{c}\text { Molecular } \\
\text { Weight (Da) }\end{array}$ & $\mathrm{pI}$ \\
\hline \multirow{10}{*}{$\begin{array}{c}\text { Pyrus } \\
\text { bretschneideri }\end{array}$} & gene 12374 & 2 & 4134 & 4 & 174 & 19594.15 & 7.72 \\
\hline & gene 14557 & 3 & 1341 & 4 & 174 & 19563.47 & 9.15 \\
\hline & gene 20820 & 5 & 1341 & 4 & 174 & 19593.50 & 9.15 \\
\hline & gene 4010 & 6 & 1748 & 4 & 192 & 21300.85 & 7.78 \\
\hline & gene7939 & 6 & 1781 & 4 & 192 & 21386.94 & 7.79 \\
\hline & gene20297 & 7 & 1173 & 4 & 173 & 19596.55 & 9.09 \\
\hline & gene 31860 & 11 & 1275 & 4 & 174 & 19643.53 & 8.86 \\
\hline & gene 23124 & 12 & 1986 & 4 & 172 & 19339.06 & 9.20 \\
\hline & rna20841 & 14 & 2677 & 4 & 172 & 19223.00 & 8.54 \\
\hline & gene 11252 & 14 & 1684 & 4 & 172 & 18872.85 & 8.79 \\
\hline \multirow{5}{*}{$\begin{array}{c}\text { Pyrus } \\
\text { communis }\end{array}$} & pycom01g20860 & 1 & 890 & 4 & 173 & 19489.22 & 8.86 \\
\hline & pycom03g09760 & 3 & 1330 & 4 & 174 & 19541.42 & 8.83 \\
\hline & pycom04g21920 & 4 & 3602 & 4 & 174 & 19566.14 & 7.71 \\
\hline & pycom07g24410 & 7 & 880 & 4 & 173 & 19566.46 & 9.09 \\
\hline & pycom $12 \mathrm{~g} 24180$ & 12 & 1658 & 4 & 174 & 19520.10 & 6.72 \\
\hline \multirow{9}{*}{$\begin{array}{c}\text { Pyrus } \\
\text { betuleafolia }\end{array}$} & GWHPAAYT001914 & 1 & 865 & 4 & 173 & 19489.22 & 8.86 \\
\hline & GWHPAAYT040350 & 4 & 4248 & 4 & 174 & 19594.15 & 7.72 \\
\hline & GWHPAAYT047043 & 6 & 1614 & 4 & 187 & 20607.89 & 6.50 \\
\hline & GWHPAAYT050130 & 7 & 885 & 4 & 173 & 19596.55 & 9.09 \\
\hline & GWHPAAYT007785 & 11 & 1275 & 4 & 174 & 19643.53 & 8.86 \\
\hline & GWHPAAYT010058 & 12 & 2130 & 4 & 172 & 19339.06 & 9.20 \\
\hline & GWHPAAYT012924 & 12 & 1934 & 4 & 174 & 19589.21 & 7.72 \\
\hline & GWHPAAYT019304 & 14 & 1648 & 4 & 172 & 18902.88 & 8.79 \\
\hline & GWHPAAYT016784 & 14 & 2402 & 4 & 172 & 19223.00 & 8.54 \\
\hline
\end{tabular}




\section{Table 2 (on next page)}

$\mathrm{Ka} / \mathrm{Ks}$ valus of $B E B P$ genes between $P$. betuleafolia and $P$. communis with $P$. betuleafolia. 


\begin{tabular}{ccccc}
\hline Seq_1 & Seq_2 & Ka & Ks & Ka/Ks \\
\hline gene20297 & pycom01g20860 & 0.058323 & 0.255761 & 0.228038 \\
gene20297 & pycom07g24410 & 0.002747 & 0.017107 & 0.160556 \\
gene4010 & GWHPAAYT019304 & 0.018274 & 0.127154 & 0.143714 \\
gene7939 & GWHPAAYT019304 & 0.069975 & 0.177029 & 0.395272 \\
gene4010 & GWHPAAYT047043 & 0.028379 & 0.033548 & 0.845944 \\
gene20297 & GWHPAAYT001914 & 0.058323 & 0.255761 & 0.228038 \\
gene20297 & GWHPAAYT050130 & 0 & 0.008547 & 0 \\
gene23124 & GWHPAAYT010058 & 0 & 0.007937 & 0 \\
gene23124 & GWHPAAYT016784 & 0.053811 & 0.100288 & 0.536559 \\
gene16540 & GWHPAAYT010058 & 0.053332 & 0.091653 & 0.581889 \\
gene11252 & GWHPAAYT019304 & 0.002585 & 0.023717 & 0.108996 \\
gene16540 & GWHPAAYT016784 & 0 & 0.007813 & 0 \\
gene11252 & GWHPAAYT047043 & 0.044601 & 0.178034 & 0.250522 \\
\hline
\end{tabular}

